\title{
Avaliação e caracterização da qualidade da carne de peito (Pectoralis major) de matrizes pesadas em final de ciclo produtivo
}

\author{
Evaluation and characterization of breast quality of broiler breeder hen in the end of productive cycle
}

\begin{abstract}
Cristiane SANFELICE ${ }^{3 *}$, Ariel Antonio MENDES ${ }^{3}$, Claudia Marie KOMIYAMA ${ }^{4}$, Marleide da Costa CAÑIZARES ${ }^{4}$, Luciana RODRIGUES ${ }^{4}$, Gil Ignácio CAÑIZARES ${ }^{4}$, Roberto de Oliveira ROÇA² ${ }^{2}$ Ibiara Correia Lima Paz ALMEIDA4, Augusto BALOG ${ }^{1}$, Elisane Lenita MILBRADT ${ }^{4}$, Karen Franco de Godoy CARDOSO ${ }^{1}$
\end{abstract}

\begin{abstract}
Resumo
Este trabalho teve por objetivo avaliar as características da qualidade da carne de matrizes pesadas de descarte. Para isto, foram coletadas 40 amostras de filés de peito (Pectoralis major) de matrizes da linhagem Ross com 68 e 69 semanas de idade. As coletas foram divididas em duas, com 20 amostras coletadas em cada uma delas. No tempo zero (após o resfriamento) foi medido o pH e coletados fragmentos para a avaliação do valor R. Nos tempos 4 e 24 horas post-mortem, foram feitas as seguintes análises: pH, valor R, cor objetiva, perda por exsudação (drip loss), capacidade de retenção de água (CRA), capacidade de absorção de água (CAA), perdas de peso por cozimento (PPC) e força de cisalhamento (FC). Houve diferença $(\mathrm{p} \leq 0,05)$ para os valores médios de $\mathrm{pH}$ entre os tempos zero (após o resfriamento), 4 e 24 horas que foram de 6,49; 5,78; e 5,65, respectivamente. O resultado médio encontrado para CRA foi de 26,45. Para a cor objetiva, os resultados médios para o $L^{*}, a^{*}$ e b* foram de 52,20; 3,64; 0,51, respectivamente. Portanto, conclui-se que, quando observados os resultados das análises para os parâmetros perda por exsudato, perda de peso por cozimento e capacidade de absorção de água, a carne de peito das matrizes pesadas apresenta ótima qualidade tecnológica apesar de apresentar problemas na sua textura.
\end{abstract}

Palavras-chave: galinha de descarte; maciez e cor; matriz pesada; pectoralis major.

\begin{abstract}
The objective of this study was to evaluate the meat quality characteristics in broiler breeder hen. For this purpose, 40 breast fillet samples (Pectoralis major) of breeder hen, Ross strain with 68 and 69 weeks of age, were collected. The samples were collected twice, and each time twenty samples were obtained. At zero time, (after chilling time) the $\mathrm{pH}$ was measured and fragmented samples were collected to evaluate the $\mathrm{R}$ value it. At 4 and 24 hours post-mortem, the following analyses were performed: $\mathrm{pH}$, R-value, color, drip loss, water holding capacity (WHC), water absorption capacity (WAC), cooking loss, and shear force. There was a significant difference ( $\mathrm{p} \leq 0.05)$ in the average $\mathrm{pH}$ values in the zero time (after chilling), 4 , and 24 hours, 6.49; 5.78, and 5.65, respectively. The average value of WHC was 26.45 . With respect to the objective color, the average values obtained for $\mathrm{L}^{\star}, \mathrm{a}^{\star}$, and $\mathrm{b}^{\star}$ were $52.20 ; 3.64 ; 0.51$; respectively. Therefore, it can be concluded that the results obtained for drip loss, cooking loss, and water absorption capacity indicate that the breast meat of breeder hens presented very good technological quality despite its texture problems.

Keywords: spend hen; tenderness and color; breeder hen; pectoralis major.
\end{abstract}

\section{Introdução}

No Brasil, nos últimos 20 anos, o número de matrizes de corte alojadas veio aumentando e, no ano de 2008 , foi registrado um total de 48,564,097. Esse número superou as previsões iniciais e teve um aumento de $14,3 \%$ sobre 2007, que apresentou um alojamento de $42,481,788$. Esse desempenho deveu-se principalmente às exportações de carne de frango e, também, ao programa de expansão das agroindústrias para atender à demanda dos mercados importadores (UBA, 2009).

Com a regulamentação e implementação do Programa de Regionalização Sanitária da Avicultura Brasileira, ficará impedido o trânsito interestadual de aves vivas em final de ciclo produtivo (ACAB, 2005), o que faz com que cada Estado seja obrigado a dar um destino adequado a estas aves que até então eram comercializadas vivas em feiras livres. Uma alternativa viável seria o abate em abatedouros comerciais, já que se tornam disponíveis para o abate no final de seu ciclo de postura.

Inicialmente, as carnes de galinhas de descarte eram utilizadas para a produção de ração animal ou no preparo de caldos concentrados, sendo apenas utilizada para consumo doméstico sob a forma de canjas e ensopados (AJUYAH et al., 1992; VOLLER-REASONOVER et al., 1997). Mas uma forma de comercialização que cresce intensamente é a venda de produtos industrializados como hambúrgueres, empanados e diversos pratos já preparados e semiprontos para o consumo. No entanto,

${ }^{1}$ Departamento de Produção Animal, Faculdade de Medicina Veterinária e Zootecnia, Universidade Estadual Paulista - UNESP, CEP 18603-970, Botucatu - SP, Brasil

2 Departamento de Gestão e Tecnologia Agroindustrial, Faculdade de Ciências Aplicadas - FCA, Universidade Estadual Paulista - UNESP, CEP 18603-970, Botucatu - SP, Brasil

${ }^{3}$ Departamento de Produção Animal, Faculdade de Medicina Veterinária e Zootecnia - FMVZ, Universidade Estadual Paulista - UNESP, CEP18618000, Botucatu - SP, Brasil, e-mail: crisanfelice@yahoo.com.br

${ }^{4}$ Faculdade de Medicina Veterinária e Zootecnia (Produção Animal) - FMVZ, Universidade Estadual Paulista - UNESP, CEP 18618-000, Botucatu - SP, Brasil

${ }^{*}$ A quem a correspondência deve ser enviada 
para a venda de partes como produtos industrializados, a sua qualidade está diretamente relacionada com a qualidade da carne utilizada no preparo. Informações sobre as propriedades físico-químicas e a forma que estas propriedades determinam a qualidade do produto final são essenciais para se obter produtos de qualidade. No entanto, existem poucos trabalhos na literatura acerca da qualidade da carne de matrizes de descarte.

Deste modo, o presente trabalho tem como objetivo avaliar e caracterizar a qualidade da carne de matrizes pesadas em final de ciclo produtivo, a fim de se conhecer suas propriedades tecnológicas para o possível uso desta carne como matéria-prima na produção de produtos industrializados de qualidade, visando agregação de valor.

\section{Material e métodos}

A obtenção das amostras para determinações das características de qualidade da carne de peito de matrizes de descarte foi realizada em linha comercial de abate. As amostras foram coletadas em abatedouro comercial localizado na cidade de Itapetininga - SP. Foram realizadas duas coletas em dias diferentes, sendo coletados 40 peitos de matrizes pertencentes à linhagem Ross com 68 e 69 semanas de idade. Em cada coleta, foram utilizadas 20 amostras de filés de peito (Pectoralis major) de matrizes pesadas de descarte.

As amostras foram coletadas de acordo com o seguinte procedimento: os peitos desossados foram coletados na sala de corte e desossa, onde foram medidos no tempo zero (após o resfriamento) os valores de $\mathrm{pH}$ e temperatura e coletados fragmentos de carne para a avaliação do valor $\mathrm{R}$. Cada peito foi embalado individualmente em sacos plásticos, devidamente identificados com anilhas plásticas, acondicionados em caixa de isopor com gelo e imediatamente transportados para o laboratório de qualidade de carne da Faculdade de Medicina Veterinária e Zootecnia - FMVZ/UNESP, campus de Botucatu. Com 4 horas post-mortem, foram novamente medidos o $\mathrm{pH}$ e valor $\mathrm{R} \mathrm{e}$, com 24 horas post-mortem, foram avaliados o $\mathrm{pH}$, temperatura, valor R, cor objetiva, perda por exsudação (drip loss), capacidade de retenção e absorção de água, perdas de peso por cozimento e força de cisalhamento.

Para as mensurações de $\mathrm{pH}$, o método utilizado foi segundo Bendall (1973), utilizando-se solução tamponada de iodoacetato de sódio $5 \mathrm{mM} / \mathrm{KCl} 150 \mathrm{mM}$, com a finalidade de inibir a glicólise. A leitura do $\mathrm{pH}$ foi realizada com pHMetro (Sentron, modelo 1001) acoplado a uma sonda (Sentron tipo LanceFET, modelo 1074001) com ponta fina de penetração, após a trituração e homogeneização por 10 segundos (Ultraturrax modelo MA 102, Marconi, Brasil) e estabilização à temperatura do homogenato a $20^{\circ} \mathrm{C}$. $\mathrm{O}$ pH nas carnes do peito de matrizes pesadas foi medido com zero (após o resfriamento), 4 e 24 horas post-mortem.

$\mathrm{Na}$ determinação do valor $\mathrm{R}$, foi feita a extração dos nucleotídeos de $2 \mathrm{~g}$ das amostras moídas, obtidas por meio da homogeneização por 30 segundos em homogeneizador com solução de ácido perclórico $1 \mathrm{M}$, proporção de 1:10 em m/v. A seguir, foi filtrado e centrifugado por 5 minutos a $3000 \mathrm{G}$. Uma alíquota de $0,1 \mathrm{~mL}$ do sobrenadante foi diluída em 4,9 mL de tampão fosfato a $0,1 \mathrm{M} \mathrm{em} \mathrm{pH} \mathrm{7,0.} \mathrm{A} \mathrm{absorbância} \mathrm{a} 250 \mathrm{~nm}$ (monofosfato de inosina) e $260 \mathrm{~nm}$ (trifosfato de adenosina) em espectrofotômetro foi determinada utilizando o tampão fosfato como referência, e o valor $\mathrm{R}$ foi determinado como razão entre as duas absorbâncias (HONIKEL; FISHER, 1977).

A avaliação da coloração da carne do peito foi determinada através de um colorímetro Minolta CR-400 (Konica Minolta), no sistema CIELab, onde foram avaliados os parâmetros $\mathrm{L}^{*}$ (luminosidade), $\mathrm{a}^{*}$ (teor de vermelho) e $\mathrm{b}^{*}$ (teor de amarelo). Os valores $\mathrm{L}^{\star}, \mathrm{a}^{\star} \mathrm{e} \mathrm{b}^{\star}$ foram medidos em três diferentes pontos na superfície ventral e no meio da seção cranial do músculo Pectoralis major. Estas avaliações foram feitas conforme metodologia proposta por Van Laack et al. (2000).

Nas medições de perda de exsudato, amostras de 100 gramas do músculo Pectoralis major foram suspensas em rede e colocadas em sacos plásticos inflados, pelo período de 48 horas à temperatura de $2{ }^{\circ} \mathrm{C}$ para a determinação da perda por exsudação, conforme metodologia descrita por Rasmussen e Anderson (1996). A determinação da porcentagem de perda por exsudação foi realizada pela diferença entre o peso final e peso inicial da amostra, conforme a Equação 1:

$\% \mathrm{PE}=(\mathrm{Pf}-\mathrm{Pi}) \times 100 / \mathrm{Pi}$

em que: $\mathrm{PE}=$ perda de exsudato; $\mathrm{Pf}=$ peso final da amostra; $\mathrm{Pi}=$ peso inicial da amostra.

A medida de capacidade de retenção de água foi realizada utilizando a metodologia descrita por Hamm (1960). A determinação foi baseada na medição da perda de água liberada quando aplicada uma pressão sobre o tecido muscular. Cubos de carne de $0,5 \mathrm{~g}$ foram colocados entre dois papéis de filtro circulares e, estes, entre duas placas de vidro, no qual é colocado peso de $10 \mathrm{~kg} / 5$ minutos. A amostra de carne de peito após a pressão foi pesada e, por diferença, calculou-se a quantidade de água perdida. O resultado foi expresso em porcentagem de água exsudada em relação ao peso inicial da amostra.

A capacidade de absorção de água (WBC) foi determinada de acordo com a metodologia proposta por Roça (1986), que consistiu em pesar exatamente $30 \mathrm{~g}$ de carne, adicionar $90 \mathrm{~mL}$ de água destilada e triturar por 90 segundos em blender. Logo após, foram pesados exatamente $35 \mathrm{~g}$ da pasta obtida em duplicata e centrifugados a $21-25^{\circ} \mathrm{C}$ por 15 minutos a $3000 \mathrm{rpm}$. O sobrenadante foi coletado e pesado e a capacidade de absorção foi calculada na Equação 2:

$\% \mathrm{WBC}=\{[(\mathrm{PP}-\mathrm{PC})-\mathrm{PS}] / \mathrm{PC}\} * 100$

em que: $\mathrm{PP}=$ peso da pasta; $\mathrm{PC}=$ peso da carne na pasta; $\mathrm{e} \mathrm{PS}=$ peso do sobrenadante.

Amostras de filés íntegros foram embaladas em papel laminado, cozidas em uma chapa metálica de dupla face, com aquecimento em ambas as faces, pré-aquecida e regulada para $180^{\circ} \mathrm{C}$, permanecendo por 4 minutos para cada lado do filé, num total de 8 minutos de cozimento ou até atingir uma temperatura interna de 82 a $85^{\circ} \mathrm{C}$. Após o cozimento, os filés foram retirados do papel laminado e resfriados sobre papel absorvente em temperatura ambiente. Posteriormente, as 
amostras foram pesadas para averiguação da quebra de peso antes e após o cozimento. A diferença entre o peso inicial (peito in natura) e final (peito cozido) correspondeu à perda de peso por cozimento (HONIKEL, 1987).

Para a avaliação da força de cisalhamento, foi utilizado o texturômetro TAXT plus equipado com dispositivo Warmer Bratzler (24 mm de altura, $8 \mathrm{~mm}$ de largura). O equipamento foi calibrado com peso padrão de $5 \mathrm{~kg}$ e padrão rastreável. A velocidade de descida do dispositivo foi de $200 \mathrm{~mm} /$ minuto (AMSA, 1995). Foram utilizadas as amostras usadas na determinação da perda de peso por cozimento. Foram retiradas 5 amostras por filé de peito na forma de paralelepípedos com $1 \times 1 \times 2 \mathrm{~cm}$ (altura, largura e comprimento, respectivamente), as quais foram colocadas com as fibras orientadas no sentido perpendicular à lâmina do probe Warner-Blatzler, e os resultados expressos em Kgf.cm ${ }^{-2}$.

Os resultados foram submetidos ao programa estatístico SAS Institute (1996), utilizando-se para a análise do pH e o valor $\mathrm{R}$ o delineamento em blocos casualizados (coletas 1 e 2) com três tempos de análise dessas características (zero, 4 e 24 horas). Para as características de qualidade, cor $\left(\mathrm{L}^{*}, \mathrm{a}^{*} \mathrm{e} \mathrm{b}^{\star}\right), \mathrm{CRA}, \mathrm{CAA}$, PE, PPC e FC, foi utilizada a estatística descritiva, sendo cada peito considerado uma repetição, totalizando 40 repetições (SAS INSTITUTE, 1996).

\section{Resultados e discussão}

A Tabela 1 mostra os resultados da estatística descritiva com valores médios, mínimos e máximos para os parâmetros de $\mathrm{pH}$ e valor $\mathrm{R}$, realizados em duas coletas diferentes ( 1 e 2), analisados na carne de peito de matrizes pesadas de descarte. Nas mensurações do $\mathrm{pH}$, os valores médio, mínimo e máximo foram de 5,97; 5,44; e 6,92, respectivamente. Em relação aos valores médios do $\mathrm{pH}$, resultados distintos foram encontrados por Jones e Grey (1989), que observaram valores médios de 5,6-5,8 em análise de peito de frangos de corte. Mas, segundo Beraquet (2000), os resultados médios de $\mathrm{pH}$ se encontram na faixa de 5,8 - 5,9. Entretanto, Qiao et al. (2001) obtiveram o valor médio de 5,96 para o $\mathrm{pH}$ medido em peito de frangos de corte, resultado semelhante ao encontrado neste trabalho para o $\mathrm{pH}$ médio medido na carne do peito das matrizes $(5,97)$.

Os valores analisados para o parâmetro valor $\mathrm{R}$ foram de 1,$24 ; 0,88 ; 1,45$ para média, mínimo e máximo, respectivamente. Entretanto, resultado médio distinto foi encontrado por Scatolini et al. (2006), que obtiveram o valor $\mathrm{R}$ de $1,65 \mathrm{em}$ amostras de peito de frangos de corte analisadas com 24 horas post-mortem.

Na Tabela 2, foram observados os parâmetros de $\mathrm{pH}$ e valor $\mathrm{R}$ para as coletas 1 e 2 mensurados com zero, 4 e 24 horas. Para as coletas 1 e 2, foram observados valores médios para o $\mathrm{pH}$ de 5,93 e 6,02, respectivamente. Deste modo, o parâmetro $\mathrm{pH}$ diferiu estatisticamente $(\mathrm{p} \leq 0,05)$ entre as coletas. Esta variação pode ter sido ocasionada devido à diferença entre a idade média das matrizes, que foi de 69 semanas de idade na primeira coleta e de 68 semanas para a segunda coleta. As medidas dos valores médios de $\mathrm{pH}$ para os tempos zero (após o resfriamento), 4 e 24 horas foram de 6,$49 ; 5,78$; e 5,65 , respectivamente, observando-se assim que houve diferença $(\mathrm{p} \leq 0,05)$ entre a medida de $\mathrm{pH}$ nos diferentes tempos. Esta queda do $\mathrm{pH}$ ocorre devido ao processo bioquímico da carne, que se prolonga após o abate, transformando a energia acumulada no músculo da ave em glicogênio lático sob a ação de várias enzimas, causando um acúmulo de ácido lático nas células, reduzindo o valor do $\mathrm{pH}$ no post-mortem.

Não foi observada diferença $(p>0,05)$ entre as duas coletas, e as medidas para o parâmetro valor $R$ entre as coletas 1 e 2 foram de 1,25 e 1,23, respectivamente. Para os diferentes tempos de análise (zero, 4 e 24 horas), as medidas do valor $\mathrm{R}$ foram de 1,09; 1,$27 ;$ e 1,42 , respectivamente, indicando que houve diferença ( $p$ $\leq 0,05)$. Esses resultados estão de acordo com Honikel e Fisher (1977), que relataram um aumento do valor $\mathrm{R}$ com o avanço do processo de rigor mortis. E ainda, segundo o constatado por Honikel et al. (1981) e sugerido por Sams e Mills (1993), o valor $\mathrm{R}$ igual ou maior do que 1,10 é utilizado como critério para saber que a ave se encontra em rigor mortis. Scatolini et al. (2006) encontraram, para análise de valor $\mathrm{R}$ realizada em peitos de frango com 5 horas post-mortem, o valor $\mathrm{R}$ de 1,27, que foi semelhante ao encontrado neste trabalho $(1,27)$ quando analisado com 4 horas post-mortem. Para a análise da interação do valor R entre a coleta e o tempo, não foi observada diferença estatística $(\mathrm{p}>0,05)$.

A Tabela 3 mostra os valores de $\mathrm{pH}$ obtidos pela interação entre as coletas ( 1 e 2) e os diferentes tempos analisados (zero, 4 e 24 horas post-mortem). Para as mensurações realizadas no tempo de análise zero, houve diferença $(\mathrm{p} \leq 0,05)$ entre as coletas 1 e 2 , sendo que o menor valor foi encontrado para a coleta 1 , com $\mathrm{pH}$ de 6,35, e o maior valor, 6,62, foi observado para a segunda coleta. As mensurações de $\mathrm{pH}$ no tempo 4 horas para as coletas 1 e 2 foram de 5,74 e 5,83, respectivamente. Porém esses resultados não diferiram $(\mathrm{p}>0,05)$. Para o $\mathrm{pH}$ medido no tempo 24 horas post-mortem, os valores encontrados nas 2 coletas foram de 5,70 e 5,61, respectivamente; portanto esses resultados não apresentaram diferença significativa $(p>0,05)$.

Tabela 1. Valores médios, mínimos e máximos para $\mathrm{pH}$ e valor $\mathrm{R}$

\begin{tabular}{lccccc}
\hline \multicolumn{1}{c}{ Análise } & Média & Mínimo & Máximo & DP & CV \\
\hline Ph & 5,97 & 5,44 & 6,92 & 0,46 & 6,95 \\
Valor R & 1,24 & 0,88 & 1,45 & 0,18 & 14,45 \\
\hline
\end{tabular}

DP: Desvio Padrão; CV: Coeficiente de Variação.

Tabela 2. Valores médios de $\mathrm{pH}$ e valor $\mathrm{R}$ para coletas 1 e 2, e mensurados nos tempos zero, 4 e 24 horas post-mortem.

\begin{tabular}{lcc}
\hline \multicolumn{1}{c}{ Parâmetros } & $\mathrm{pH}$ & Valor R \\
\hline \multicolumn{1}{c}{ Coletas } \\
2 & 5,93 & \\
& 6,02 & 1,25 \\
& Tempo & 1,23 \\
Zero & 6,49 & \\
4 horas & 5,78 & $1,09 \mathrm{~b}$ \\
24 horas & 5,65 & $1,27 \mathrm{ab}$ \\
& Probabilidade & $1,42 \mathrm{a}$ \\
Coleta & 0,0052 & \\
Tempo & $<0,0001$ & 0,26 \\
Coleta $\times$ Tempo & $<0,0001$ & $<0,0001$ \\
& & 0,1357
\end{tabular}


Tabela 3. Valores médios encontrados para o desdobramento da interação do $\mathrm{pH}$ entre tempos de análise e coletas 1 e 2 da carne de peito de matrizes pesadas de descarte.

\begin{tabular}{lcc}
\hline Tempo & \multicolumn{2}{c}{ Coletas } \\
\hline & 1 & 2 \\
\hline Zero & $6,35^{\mathrm{aB}}$ & $6,62^{\mathrm{aA}}$ \\
4 horas & $5,74^{\mathrm{b}}$ & $5,83^{\mathrm{b}}$ \\
24 horas & $5,70^{\mathrm{c}}$ & $5,61^{\mathrm{c}}$ \\
\hline
\end{tabular}

Médias seguidas por letras minúsculas na coluna e maiúsculas na linha diferem entre si $(\mathrm{p} \leq 0,05)$ pelo teste de Tukey.

Ainda de acordo com a Tabela 3, os valores encontrados na primeira coleta para as medições do $\mathrm{pH}$ nos tempos zero, 4 e 24 horas post-mortem foram de 6,35; 5,74; e 5,70, respectivamente. Dessa forma, esses valores apresentaram diferença $(\mathrm{p} \leq 0,05)$ entre si.

Os resultados encontrados na coleta 2 para os três diferentes tempos foram de 6,$62 ; 5,83$; e 5,61, respectivamente, observando-se assim que houve diferença estatística $(\mathrm{p} \leq 0,05)$ entre os diferentes tempos. Os maiores valores de $\mathrm{pH}$ encontrados foram obtidos nas medições do tempo zero para ambas as coletas ( 1 e 2). Da mesma forma, os menores valores de $\mathrm{pH}$ foram observados (nas duas coletas) para o tempo de análise 24 horas post-mortem. Estes resultados estão relacionados à queda do $\mathrm{pH}$, conforme ocorre um aumento no tempo post-mortem da carne.

Na Tabela 4, encontram-se os resultados médios, mínimos e máximos para os parâmetros: cor objetiva $\left(L^{*}, a^{*}\right.$ e $\left.b^{*}\right)$, perda de exudato (drip loss), perda de peso por cozimento, capacidade de retenção e absorção de água e força de cisalhamento. Para o parâmetro luminosidade, a média dos valores foi de 52,20, os valores mínimo e máximo encontrados foram de 43,80 e 59,59, respectivamente. Porém, resultados médios distintos foram encontrados por Sams e Dzuik (1999) para o valor $L^{\star}(54,86)$ em medições realizadas com 24 horas post-mortem para carne de peito de frangos de corte. Valores muito elevados de $L^{*}$ não são bons, pois indicam um aumento na palidez da carne, o que influencia diretamente o consumidor no momento da compra.

O parâmetro $a^{\star}$ apresentou os valores de 3,64; 1,21; e 8,70 como sendo média, mínimo e máximo, respectivamente. Porém resultados distintos foram encontrados por Qiao et al. (2002), que obtiveram o valor de 4,38 para o teor de vermelho $\mathrm{a}^{*}$ em análises da carne de peito de frangos de corte. $\mathrm{O}$ teor de amarelo $b^{\star}$ obteve para as análises de média, mínimo e máximo os valores 0,$51 ;-3,59$; e 3,78, respectivamente.

$\mathrm{Na}$ análise estatística do parâmetro capacidade de retenção de água (CRA) os resultados encontrados para média, mínimo e máximo foram de 26,45; 15,00; e 35,00, respectivamente. Entretanto, resultados distintos foram encontrados por Qiao et al. (2001), que obtiveram o valor médio de 43,77, em análise realizada em peitos de frangos de corte.

As mensurações feitas para o parâmetro capacidade de absorção de água (CAA) encontraram para média, mínimo e máximo os valores de 64,54; 26,86; e 88,57, respectivamente.
Tabela 4. Valores médios, mínimos e máximos para medidas de cor objetiva $\left(L^{*}, a^{*}\right.$ e $\left.b^{\star}\right)$, capacidade de retenção (CRA) e absorção de água (CAA), perda por exsudação (PE) (drip loss), perdas por cocção (PPC) e força de cisalhamento (FC) da carne de matrizes pesadas de frango de corte.

\begin{tabular}{crrrrr}
\hline Parâmetros & Média & Mínimo & Máximo & \multicolumn{1}{c}{ DP } & \multicolumn{1}{c}{ CV } \\
\hline $\mathrm{L}^{*}$ & 52,20 & 43,80 & 59,59 & 3,11 & 5,95 \\
$\mathrm{a}^{*}$ & 3,64 & 1,21 & 8,70 & 1,61 & 44,34 \\
$\mathrm{~b}^{*}$ & 0,51 & $-3,59$ & 3,78 & 1,91 & 375,72 \\
$\mathrm{CRA}$ & 26,45 & 15,00 & 35,00 & 4,77 & 18,04 \\
$\mathrm{CAA}$ & 64,54 & 26,86 & 88,57 & 12,98 & 20,11 \\
PE & 1,64 & 1,13 & 2,32 & 0,27 & 16,46 \\
PPC & 22,02 & 13,60 & 36,18 & 4,75 & 21,55 \\
FC & 5,80 & 3,54 & 10,3 & 165 & 28,45 \\
\hline Sendo, DP. desvio padrão; CV: coeficiente de variação.
\end{tabular}

Entretanto, Moreira (2005) encontrou resultado distinto para esse parâmetro em frangos de corte obtendo valor médio de 33,66 .

O parâmetro perda de exsudato obteve os valores 1,64 ; 1,13; e 2,32 para as análises média, mínimo e máximo, respectivamente. Porém, foi relatado por Moreira (2005) valor médio de 2,19 (mais elevado que o encontrado nesta coleta) em análise feita em peito de frangos de corte.

As análises de perda de peso por cocção (PPC) apresentaram para média, mínimo e máximo os valores de 22,02; 13,60; e 36,18 , respectivamente. Esses resultados diferem dos encontrados por Scatolini et al. (2006), que obtiveram o valor médio de 24,79 em análise realizada em peitos de frango com 24 horas post-mortem.

As medições feitas para a análise da força de cisalhamento resultaram em 5,80, 3,54, 10,3 como sendo os valores médio, mínimo e máximo obtidos. Entretanto Moreira et al. (2004), analisando a carne de peitos de frangos de corte encontraram resultados médios de 3,94 para a força de cisalhamento. Resultado distinto também foi encontrado por Canizares (2008), que obteve o valor médio de 2,02 para este parâmetro na carne de peito de frango de corte embalada a vácuo e mantida sob refrigeração. E ainda, Allen et al. (1998), que obtiveram o valor médio de 3,49 para a FC quando medidos peitos de frangos de corte.

\section{Conclusões}

Neste trabalho com a carne de peito de matrizes pesadas, conclui-se que, quando observados os resultados das análises para os parâmetros perda por exsudato, perda de peso por cozimento e capacidade de absorção de água, a carne de peito das matrizes pesadas apresenta qualidade tecnológica comparável e até superior à carne de frango. Embora ainda sejam necessários alguns estudos e métodos para a obtenção de uma carne mais macia, esta carne pode ser utilizada como matéria-prima na produção de produtos industrializados de qualidade. 


\section{Referências bibliográficas}

ASSOCIAÇÃO DOS CRIADORES DE AVESTRUZES DO BRASIL ACAB. Programa de regionalização sanitária da avicultura brasileira. São Paulo, 2005. Disponível em: <www.acab.org.br/ download/Proposta_Definitiva_de_Regionalizacao.doc $>$. Acesso em: 03 set. 2005.

AJUYAH, A. O. et al. Yield, lipid cholesterol and fatty acid composition of spent hens full-fat oil seeds and fishmeal diets. Journal of Food Science, v. 57, n. 2, p. 338-341, 1992.

ALLEN, C. D. et al. The relationship of broiler breast color to meat quality and shelf-life. Poultry Science, v. 77, n. 2, p. 361-366, 1998.

AMERICAN MEAT SCIENCE ASSOCIATION - AMSA. Research guideliness for cookery sensory and instrumental tenderness measurement of fresh meat. Chicago, 1995. $48 \mathrm{p}$.

BENDALL, J. R. Post-mortem change in muscle. In: BOURNE, G. H. (Ed.). The structure and function of muscle. New York: Academic Press, 1973. p. 242-309.

BERAQUET, N. J. Carne mecanicamente separada de aves. In: SEMINÁRIO E CURSO TEÓRICO-PRÁTICO "AGREGANDO VALOR A CARNE DE AVES”, 2000, Campinas. Anais... Campinas: ITAL, 2000. p. 17-19.

CANIZARES, M. C. Qualidade da carne de frango submetida à irradiação ou atmosfera modificada e armazenada por diferentes períodos. 2008. 93 p. Tese (Doutorado) - Universidade Estadual Paulista - UNESP, Botucatu.

HAMM, R. Biochemistry of meat hydratation: advances in food research. Cleveland, v. 10, n. 2, p. 335-443, 1960.

HONIKEL, K. O.; FISHER C. A. A rapid method for the detection of PSE and DFD porcine muscles. Journal of Food Science, v. 42, n. 7, p. 1663-1676, 1977.

HONIKEL, K. O. et al. Influence of post mortem changes in bovine muscle on the water-holding capacity of beef: post mortem storage of muscle at $20^{\circ} \mathrm{C}$. Journal of Food Science, v. 46 , n. 1, p. 1-7, 1981.

HONIKEL, K. O. Influence of chilling on meat quality attributes of fast glycolysing pork muscles. In: TARRANT, P. V.; EIKELENBOOM, G.; MONIN, G. (Eds.). Evaluation and control of meat quality in pigs. Dordrecht: Martinus Nijhoff, 1987. p. 273-283.

JONES, J. M.; GREY, T. C. Influence of processing on product quality and yield. In: MEAD, G. C. (Ed.). Processing of poultry. London: Chapman \& Hall, 1989. p. 127-130.
MOREIRA, J. Causas da ocorrência de carne PSE em frangos de corte e como controlá-las. In: SEMINÁRIO INTERNACIONAL DE AVES E SUÍNOS, 4, 2005, Florianópolis. Anais...

MOREIRA, J. et al. Efeito da densidade populacional sobre desempenho, rendimento de carcaça e qualidade da carne em frangos de corte de diferentes linhagens comerciais. Revista Brasileira de Zootecnia, v. 33, n. 6, p. 1506-1519, 2004.

QIAO, M. et al. The relationship between raw broiler breast meat color and composition. Poultry Science, v. 81, n. 3, p. 422-427, 2002.

QIAO, M. et al. The effect of broiler breast meat color on $\mathrm{pH}$, moisture, water-holding capacity, and emulsification capacity. Poultry Science, v. 80, n. 5, p. 676-680, 2001.

RASMUSSEN, A.; ANDERSSON, M. New methods for determination of drip loss in pork muscles. In: INTERNATIONAL CONGRESS OF MEAT SCIENCE AND TECHNOLOGY, 42, 1996, Lillehammer. Proceedings... Matforsk, Lillehammer, Norway, 1996. p. 286-287.

ROÇA, R. O. Desenvolvimento de fiambres com carne de frango. 1986. 183 p. Dissertação (Mestrado em Engenharia de Alimentos) Universidade Estadual de Campinas - UNICAMP, Campinas.

SAMS, A. R.; MILLS, K. A. The effect of feed withdrawal duration on the responsiveness of broiler Pectoralis to rigor mortis acceleration. Poultry Science, v. 72, n. 9, p. 1789-96, 1993.

SAMS, A. R.; DZUIK, C. S. Meat quality and rigor mortis development in broiler chickens with gas-induced anoxia and postmortem electrical stimulation. Poultry Science, v. 78, n. 10, p. 1472-1476, 1999.

SAS INSTITUTE. SAS use's guide. Cary, 1996.

SCATOLINI, A. M. et al. Efeito do período de desossa e do tempo de armazenamento em refrigeração na qualidade da carne de peito de frangos. Revista Portuguesa de Ciências Veterinárias, v. 101, n. 559-560, p. 257-262, 2006.

UNIÃO BRASILEIRA DE AVICULTURA - UBA. Alojamento de matriz de corte: relatório anual 2008. São Paulo, 2009. p. 24. Disponível em: <http://www.uba.org.br/site3/arquivos/anuario_ uba_2008_port_site.pdf>. Acesso em: 26 jun. 2009.

VAN LAACK, R. L. J. M. et al. Characteristics of pale, soft, exudative broiler breast meat. Poultry Science, v. 79, n. 7, p. 1057-1061, 2000.

VOLLER-REASONOVER, L. et al. High temperature processing effects on the proprieties of fowl meat gels. Poultry Science, v. 76, n. 6, p. 774-779, 1997. 